\title{
Rapid Formation of Cerebral Microbleeds after Carotid Artery Stenting
}

\author{
Kousuke Kakumoto $^{\text {a }}$ Shoji Matsumoto ${ }^{\text {a-c }}$ Ichiro Nakahara ${ }^{a}$ \\ Yoshihiko Watanabe $^{\text {a }}$ Yutaka Fukushima $^{\text {a }}$ Urabe Yoshikiyo $^{a}$ \\ Ryota Ishibashia Masanori Gomi ${ }^{a}$ Keiichi Tsuji ${ }^{a}$ \\ Yoshinori Sanbongi ${ }^{a}$ Tetsuya Hashimoto ${ }^{a}$ Yujiro Tanaka ${ }^{a}$ \\ Takeshi Yamada ${ }^{b}$ Jun-ichi Kirac \\ ${ }^{a}$ Department of Neurosurgery, Kokura Memorial Hospital, ${ }^{b}$ Department of Neurology, \\ Saiseikai General Hospital, and ' ${ }^{\mathrm{C}}$ Department of Neurology, Neurological Institute, Graduate \\ School of Medical Sciences, Kyushu University, Fukuoka, Japan
}

\section{Key Words}

Cerebral microbleeds $\cdot$ Carotid artery stenting $\cdot$ MRI

\begin{abstract}
Background: Recent studies reported that cerebral microbleeds (CMBs), i.e. small areas of signal loss on $\mathrm{T}_{2}{ }^{*}$-weighted gradient-echo (GE) imaging, could develop rapidly after acute ischemic stroke. We hypothesized that CMBs rapidly emerge after carotid artery stenting (CAS). Objective: We investigated the frequency of and predisposing factors for CMBs after CAS. Methods: We retrospectively examined MRI before and after CAS in 88 consecutive patients (average age: $71.7 \pm 7.2$ years, average rates of carotid stenosis: $72.6 \pm 12.8 \%$ ) who underwent CAS for carotid artery stenosis between March 1, 2009, and September 30, 2010. We defined new CMBs as signal losses that newly appeared on the follow-up GE. We examined the association of new CMBs with demographics, risk factors, and baseline MBs. Results: Among 88 patients, $18(20.5 \%)$ had CMBs initially, and 7 (8.0\%) developed new CMBs right after CAS. New CMBs appeared on the same side of CAS in all of the 7 patients. New CMBs appeared significantly more frequently
\end{abstract}

K.K. and S.M. contributed equally to this work.

Shoji Matsumoto, MD

Department of Neurology, Fukuoka Saiseikai General Hospital

1-3-46 Tenjin, Chuo-ku

Fukuoka 810-0001 (Japan)

Tel. +81 92771 8151, E-Mail shoji-matsumoto@ 384.jp 


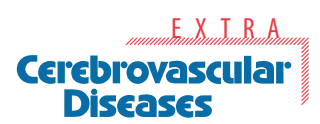

Cerebrovasc Dis Extra 2012;2:9-16

DOI: $10.1159 / 000337143$

Published online: March 14, 2012

Kakumoto et al.: Rapid Formation
๑) 2012 S. Karger AG, Basel

www.karger.com/cee

in the CMB-positive group than in the CMB-negative one ( $22 \%$ vs. $4 \%, \mathrm{p}=0.03)$ on the pre-CAS MRI. Multivariate analysis also revealed that the presence of CMBs before CAS was an independent predictor of new development of CMBs after CAS (odds ratio: 8.09, 95\% confidence interval: 1.39-47.1). Conclusion: CMBs can develop rapidly after CAS, especially in patients with preexisting CMBs. Since the existence of CMBs prior to CAS suggests a latent vascular damage which is vulnerable to hemodynamic stress following CAS, particular attention should be paid to the prevention of intracerebral hemorrhage due to hyperperfusion after CAS.

Copyright $\odot 2012$ S. Karger AG, Basel

\section{Introduction}

Cerebral microbleeds (CMBs) are focal hemosiderin deposits adjacent to small vessels resulting from minimal blood leakage from small vessels that appear as signal loss lesions on $\mathrm{T}_{2}{ }^{*}$-weighted gradient-echo (GE) magnetic resonance imaging (MRI). CMBs can be regarded as a marker of microangiopathy $[1,2]$. A recent review of the literature suggests that there is a strong association between CMBs identified on MRI and histopathological evidence of previous hemorrhage, most commonly in the form of hemosiderin-laden macrophages [3]. Many studies suggested that CMBs could be associated with an increased risk of intracerebral hemorrhage ( $\mathrm{ICH}$ ) and cerebral amyloid angiopathy [4-7] and can develop rapidly after acute ischemic stroke [8].

Cerebral hyperperfusion after carotid artery stenting (CAS) and carotid endarterectomy (CEA) is well known, and is defined as a marked increase in ipsilateral cerebral blood flow following surgical repair of carotid stenosis that results in a risk of ICH [9-17]. We hypothesized that CMBs may develop rapidly after CAS. This study investigated the frequency and predisposing factors of new $\mathrm{CMB}$ formation after CAS.

\section{Methods}

\section{Patients}

We retrospectively examined 88 consecutive patients who underwent CAS for carotid stenosis and MRI before and after CAS between March 1, 2009, and September 30, 2010, in Kokura Memorial Hospital. The preoperative degree of carotid stenosis was evaluated based on the North American Symptomatic Carotid Endarterectomy Trial (NASCET) criteria [18]. Clinical presentation was classified as symptomatic or asymptomatic. Symptomatic carotid stenosis was defined as either a transient ischemic attack or a nondisabling stroke on the side ipsilateral to CAS within 6 months before CAS. This study was approved by our Institutional Review Board. We have obtained informed consent from the patients.

\section{Magnetic Resonance Imaging}

MRI examinations were performed before and one day after CAS with a 1.5-T magnetic resonance system using a standard head coil. The gradient recalled echo (GRE) parameters were as follows: repetition time $=700 \mathrm{~ms}$, echo time $=20 \mathrm{~ms}$, flip angle $=25$, matrix $=288$ $\times 256$, field of view $=220 \times 220$, slice thickness $=5 \mathrm{~mm}$, and interslice gap $=2 \mathrm{~mm}$. Patients with probable cerebral amyloid angiopathy according to the Boston criteria were excluded. The presence of CMBs on GRE MRI was independently evaluated by 2 neurologists (S.M, K.K) blind to the clinical details. CMBs were defined as focal areas of very low signal intensity, with a diameter smaller than $10 \mathrm{~mm}$. The number and location of CMBs were assessed. The location of CMBs were classified as (1) lobar: in the cortex, subcortex, and white matter 
of frontal, temporal, parietal, and occipital lobes; or (2) deep or infratentorial: in the caudate, putamen, globus pallidus, internal capsule, thalamus, brainstem, and cerebellum. Hypointense lesions within the subarachnoid space were regarded as pial blood vessels. Symmetric hypointense lesions in the area of the globus pallidus were regarded as calcification. We aligned the GRE slices of the pre- and post-CAS MRI to guarantee a precise comparison. When CMBs were identified on the post-CAS GRE, their prior absence was confirmed by re-examining the corresponding pre-CAS GRE.

Technetium-99m Hexamethylpropyleneamine Oxime SPECT

Technetium-99m hexamethylpropyleneamine oxime ( $\left.{ }^{99 \mathrm{~m}} \mathrm{Tc}-\mathrm{HMPAO}\right)$ SPECT was performed the next day after CAS. Patients received $15 \mathrm{mCi}(555 \mathrm{MBq}){ }^{99 \mathrm{~m}} \mathrm{Tc}-\mathrm{HMPAO}$, and the first scan for cerebral blood flow imaging was started 5 min later (acquisition time: $20 \mathrm{~min}$ ). The images were reconstructed in a $128 \times 128$ matrix with a section thickness of $5 \mathrm{~mm}$ on the transverse, sagittal, and coronal planes. An irregular, mirror-shaped region of interest was placed manually and bilaterally over the whole middle cerebral artery territory at the level of the parietal lobe, excluding the infarct, and the corresponding contralateral region. The asymmetry index (AI; affected side counts per pixel/contralateral side counts per pixel $\times 100 \%$ ) was calculated. In the present study, hyperperfusion after CAS was defined as an AI increase of $>6.1 \%$ as described previously [19].

\section{Clinical Assessments}

The diagnosis of hypertension was based on either the use of antihypertensives or a systolic blood pressure $>140 \mathrm{~mm} \mathrm{Hg}$ and/or diastolic blood pressure $>90 \mathrm{~mm} \mathrm{Hg}$ during two separate measurements after the acute stroke period. The diagnosis of diabetes mellitus was based on the use of antidiabetic treatment or HbAlc $>6.1 \%$. Current smoking was assigned to persons who smoked during the 3 months preceding the last stroke event. Hyperlipidemia was determined when total cholesterol was $>210 \mathrm{mg} / \mathrm{dl}$ or low-density lipoprotein cholesterol was $>130 \mathrm{mg} / \mathrm{dl}$. Demographics including age, sex, and stroke risk factors were obtained.

Cerebral hyperperfusion syndrome (CHS) after CAS was diagnosed according to the following criteria: (1) severe headache, seizure, deterioration of consciousness level or the development of focal neurological signs such as motor weakness; (2) absence of any additional ischemic lesion on CT or MRI; (3) an increase in AI by $>6.1 \%$.

\section{Procedural Techniques}

All patients were pretreated with aspirin (100 mg/day) and clopidogrel (75 mg/day), or cilostazol (200 mg/day) for at least 3 days before the procedure. Eight patients also took warfarin for complicating atrial fibrillation in addition to antiplatelet drugs. Carotid angioplasty and stent placement were performed by transfemoral catheterization under local anesthesia. The distal protection of a Percusurge Guard Wire System (Medtronic AVE, Santa Rosa, Calif., USA) or EZ filter System (Boston Scientific, Natick, Mass., USA) was used to cross and pre-dilate the stenosis. Pre-dilatation was performed with a controlled-compliant balloon dilation catheter to achieve a minimal lumen for passage of the stent-mounted catheter. The balloon was inflated with 6-10 atm for $30 \mathrm{~s}$. Next, a self-expanding stent was deployed. The stent system used in this series consisted of a Precise (Johnson and Johnson, Miami Lakes, Fla., USA) in 55 cases, a Wallstent RP (Boston Scientific) in 29 cases, and a Driver (Medtronic Inc., Santa Rosa, Calif., USA) in 4 cases. Post-stenting dilatation was performed with a controlled compliant balloon dilation catheter. The balloon size was selected according to the normal luminal diameter of each internal carotid artery just distal to the stenotic segment (diameter at full dilation was usually $4.0-5.0 \mathrm{~mm}$ ). The balloon was in- 
flated with 6-10 atm for 10-30 s. Intravenous heparin was administered during the procedure, which was controlled to an activated clotting time (ACT) of around $300 \mathrm{~s}$ and not reversed at completion. A systolic blood pressure of $<140 \mathrm{~mm} \mathrm{Hg}$ was maintained for two days after the procedure. For patients at high risk of CHS, we strictly controlled blood pressure to $<120 \mathrm{~mm} \mathrm{Hg}$ for two days after CAS. Because the carotid sinus reflex usually lowered blood pressure after CAS, intravenous catecholamine was occasionally administered to maintain blood pressure.

\section{Statistical Analysis}

Student's t test was used to analyze continuous data, and the $\chi^{2}$ test was used for categorical data. Multiple logistic regression analysis was performed to estimate the independent predicting factors for the development of new CMBs after CAS. Variables with a p value $<0.1$ by univariate analysis, age, and risk factors were selected for entry into multiple regression analysis. A 2-tailed p value $<0.05$ was considered a significant difference. SPSS for Windows (version 13.0, SPSS Inc., Chicago, Ill., USA) was used for all statistical analyses.

\section{Results}

A total of 100 patients underwent CAS for intracranial artery stenosis in our hospital during the study period, 12 patients did not undergo GRE MRI. Therefore, we analyzed 88 patients (average age: $71.7 \pm 7.2$ years, male: $86 \%, 40$ symptomatic and 48 asymptomatic cases, average rates of carotid stenosis: $72.6 \pm 12.8 \%)$. Carotid stenosis was successfully treated in all patients. No patients had CHS. Demographics of the enrolled patients are shown in table 1. Eighteen patients (20.5\%) had CMBs at baseline. The median number of baseline CMBs was 2 (range: 1-5). On the follow-up MRI, we observed 8 new CMBs ipsilateral to CAS in 7 patients $(8.0 \%)$. The median number of new CMBs among these patients was 1 (range: 1-2). There were no baseline CMBs in 3 patients who developed new CMBs. A representative case with new CMBs after CAS is shown in figure 1.

By univariate analysis, the frequency of new $\mathrm{CMB}$ appearance was significantly higher in the initially CMB-positive group than the CMB-negative group (22\% vs. $4 \%, \mathrm{p}=0.03)$. There was no significant association between the appearance of new CMBs and anti-thrombotic therapy or the highest ACT values during CAS procedure. No patients developed CHS after CAS (table 2).

Multivariate analysis adjusted for age and vascular risk factors revealed that the presence of CMBs before CAS was an independent predictor of the appearance of new CMBs after CAS (odds ratio: 8.50, 95\% confidence interval: 1.34-53.88; table 3).

\section{Discussion}

This study showed that (1) CMBs can develop rapidly after CAS, and (2) the presence of CMBs before CAS was an independent predictor of the appearance of new CMBs after CAS. A recent study suggested that the presence of $\mathrm{CMBs}$ and severe small vessel diseases are predictors of the rapid development of CMBs after acute ischemic stroke [8]. Hypertension is associated with CMBs in patients with stroke [20]. In our study, risk factors for stroke, including hypertension, were not associated with the formation of new CMBs after CAS. Such risk factors may be important promoters of CMBs over a longer period but may not contribute to CMBs in the short term. The mechanism underlying the rapid formation of CMBs after CAS remains unknown. We speculate that hemodynamic changes caused by the CAS 
Table 1. Univariate factors for the development of new CMBs

\begin{tabular}{|c|c|c|c|}
\hline & \multicolumn{3}{|l|}{ New CMBs } \\
\hline & present $(n=7)$ & absent $(\mathrm{n}=81)$ & $\mathrm{p}$ value \\
\hline \multicolumn{4}{|l|}{ Demographics } \\
\hline Age & $74.0 \pm 7.9$ & $71.5 \pm 7.1$ & 0.37 \\
\hline Male sex & $7(100 \%)$ & $69(85 \%)$ & 0.59 \\
\hline Symptomatic clinical presentation & $4(57 \%)$ & $36(44 \%)$ & 0.70 \\
\hline \multicolumn{4}{|l|}{ Angiographic findings } \\
\hline Carotid stenosis (NASCET criteria), \% & $73.9 \pm 15.0$ & $72.5 \pm 12.7$ & 0.79 \\
\hline \multicolumn{4}{|l|}{ MRI findings } \\
\hline Presence of CMBs before CAS on GRE & $4(57 \%)$ & $14(17 \%)$ & 0.03 \\
\hline \multicolumn{4}{|l|}{ Risk factors } \\
\hline Hypertension & $4(57 \%)$ & $60(74 \%)$ & 0.38 \\
\hline Diabetes & $3(43 \%)$ & $26(32 \%)$ & 0.68 \\
\hline Hyperlipidemia & $3(43 \%)$ & $33(41 \%)$ & 1.00 \\
\hline Ischemic heart disease & $4(57 \%)$ & $23(28 \%)$ & 0.19 \\
\hline \multicolumn{4}{|l|}{ Laboratory findings } \\
\hline Platelet count, $\times 10,000 / \mathrm{ml}$ & $21.6 \pm 12.7$ & $20.4 \pm 5.9$ & 0.81 \\
\hline \multicolumn{4}{|l|}{ Antithrombotic therapy } \\
\hline \multicolumn{4}{|l|}{ Antiplatelet drug } \\
\hline Dual therapy (aspirin + clopidogrel) & $4(57 \%)$ & $53(65 \%)$ & \\
\hline Triple therapy (aspirin + clopidogrel + cilostazol) & $2(27 \%)$ & $20(25 \%)$ & \\
\hline \multicolumn{4}{|l|}{ Antiplatelet drug + anticoagulation drug } \\
\hline Dual antiplatelet drug + warfarin & 0 & $5(6 \%)$ & \\
\hline Triple antiplatelet drug + warfarin & $1(14 \%)$ & $3(4 \%)$ & 0.55 \\
\hline The highest ACT values during CAS procedure, $s$ & $338.9 \pm 55.0$ & $340.8 \pm 57.3$ & 0.93 \\
\hline
\end{tabular}

Values are number (\%), or mean \pm SD. p values are calculated using Student's $t$ test and the $\chi^{2}$ test.

Table 2. Demographics of patients with newly developed CMBs

\begin{tabular}{lllllll}
\hline Case No. & $\begin{array}{l}\text { Age } \\
\text { years }\end{array}$ & $\begin{array}{l}\text { Stenosis } \\
\text { (NASCET } \\
\text { criteria), } \%\end{array}$ & $\begin{array}{l}\text { CHS after } \\
\text { CAS }\end{array}$ & $\begin{array}{l}\text { CMBs before } \\
\text { CAS, n }\end{array}$ & $\begin{array}{l}\text { CMBs after } \\
\text { CAS, n }\end{array}$ & $\begin{array}{l}\text { New CMB } \\
\text { hemisphere }\end{array}$ \\
\hline 1 & 68 & 56 & no & 1 & 3 & ipsilateral \\
2 & 84 & 78 & no & 1 & 2 & ipsilateral \\
3 & 77 & 80 & no & 0 & 1 & ipsilateral \\
4 & 79 & 53 & no & 0 & 1 & ipsilateral \\
5 & 73 & 70 & no & 0 & 1 & ipsilateral \\
6 & 77 & 90 & no & 1 & 2 & ipsilateral \\
7 & 60 & 90 & no & 1 & 2 & \\
\hline
\end{tabular}

procedure affect damaged small cerebral vessels in patients with CMBs, and may disrupt the tight junction of cerebral blood vessels, resulting in CMBs.

Several studies reported that low platelet counts were associated with symptomatic hemorrhagic transformation in acute ischemic stroke with atrial fibrillation [21] and a high risk of intracranial hemorrhage after intravenous recombinant tissue plasminogen activator 


\section{Cerebirovascular $X$ II R A
Diseases}

Fig. 1. A representative case developing new CMBs after CAS. $\mathrm{T}_{2}{ }^{*}$-weighed GE MRI obtained in a 68-year-old man with symptomatic left internal carotid artery stenosis (56\%) before CAS (a, b) and the next day after CAS (c, d). Arrowheads indicate baseline CMBs. Arrows indicate new CMBs.

Table 3. Multiple logistic regression analysis of the development of new CMBs

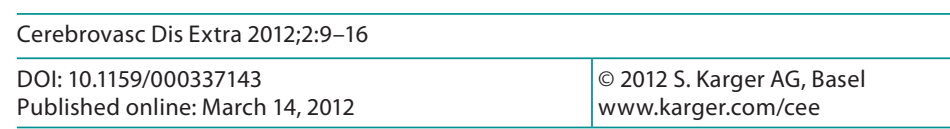

Kakumoto et al.: Rapid Formation of Cerebral Microbleeds after Carotid Artery Stenting
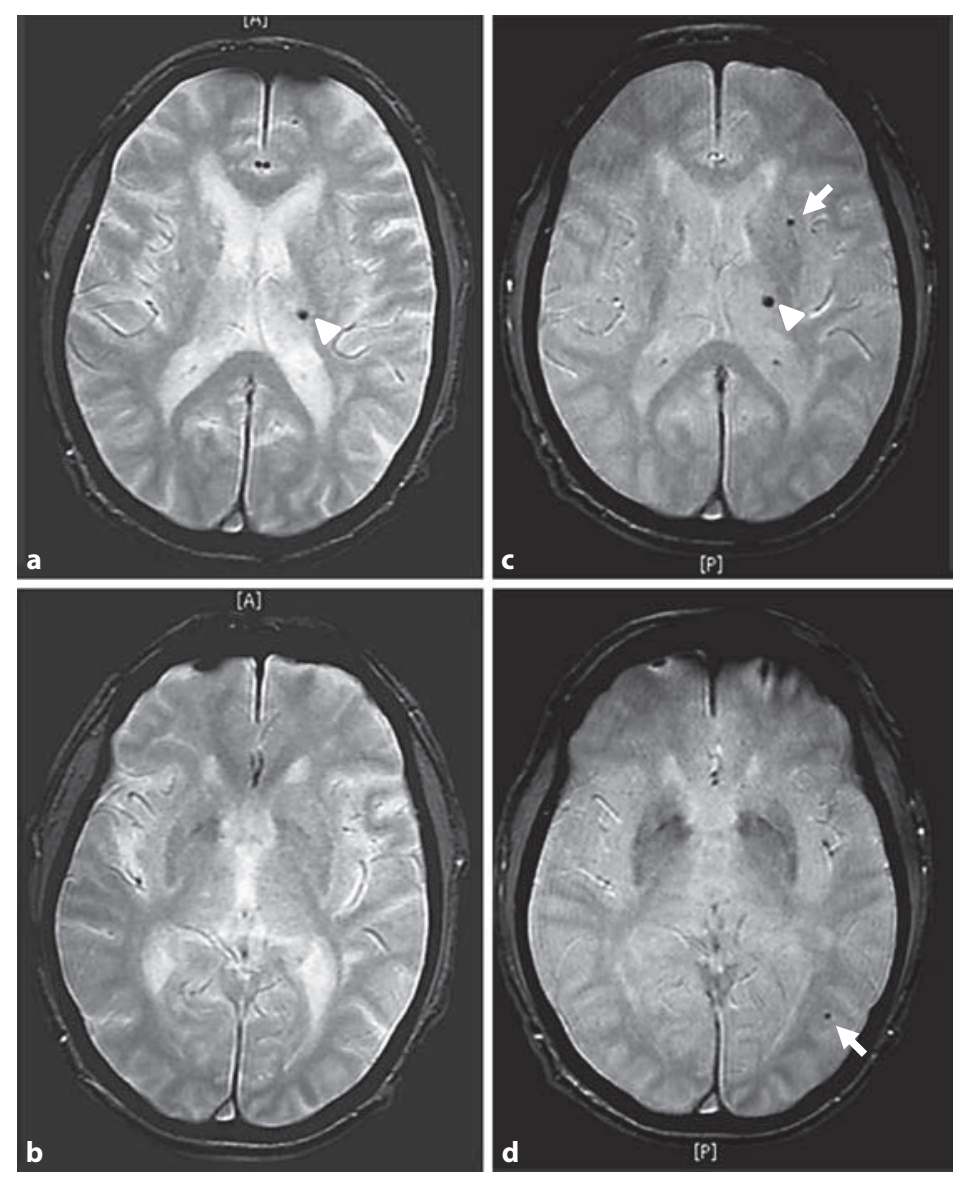

\begin{tabular}{llll}
\hline & OR & $95 \%$ CI & p value \\
\hline Presence of CMBs before CAS & 8.09 & $1.39-47.13$ & 0.02 \\
Age & 1.08 & $0.96-1.21$ & 0.20 \\
Hypertension & 0.26 & $0.04-1.80$ & 0.17 \\
Hyperlipidemia & 1.31 & $0.22-7.63$ & 0.77 \\
Diabetes & 2.46 & $0.32-19.10$ & 0.39 \\
Ischemic heart disease & 3.10 & $0.49-19.48$ & 0.23 \\
\hline
\end{tabular}

$\mathrm{OR}=$ odds ratio; $\mathrm{CI}$ = confidence interval.

therapy for acute ischemic stroke [22]. There was no association between platelet counts and the development of new CMBs after CAS in our study.

Dual antiplatelet therapy with clopidogrel and aspirin has been recommended as a standard therapy for the prevention of thrombotic events in patients undergoing CAS and intracranial artery stenting [23]. All of the patients were treated with at least two antiplatelet drugs, aspirin and clopidogrel, and some patients were also administered cilostazol and/or warfarin. Since we did not find that the development of new CMBs after CAS was associated with antithrombotic therapy, strong antithrombotic therapy during the periprocedural period may not be contraindicated by the presence of CMBs. 


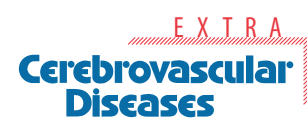

Diseases
Cerebrovasc Dis Extra 2012;2:9-16

DOI: $10.1159 / 000337143$
Published online: March 14, 2012

Kakumoto et al.: Rapid Formation of Cerebral Microbleeds after Carotid Artery Stenting

CHS is an uncommon but devastating complication following CAS and CEA, and is often fatal once cerebral hemorrhage occurs [9-17]. Before a CAS/CEA procedure, it is very important to evaluate the risks of cerebral hemorrhage in advance. CMBs may be a risk factor for ICH after CAS, and the development of new CMBs may also enhance this risk. Although we had no patients with CHS after CAS in this series, we should pay special attention to the development of CHS in case of preexisting CMBs.

Our study has some limitations. First, although we used defined MRI protocols for all patients before and after CAS, our study has a retrospective design and may have a potential risk for selection bias. It remains unknown whether CMBs are more frequent in patients with severe atherosclerotic changes in the carotid artery who undergo CAS than in those who do not require CAS. Second, this study is limited by its small sample size, and we could not completely evaluate several factors. Third, we used 1.5-T MRI, which is inferior to 3-T MRI for the detection of CMBs. The use of 3-T MRI might have allowed the detection of more CMBs [24].

Our study suggests that new CMBs can occur rapidly after CAS. The presence of baseline $\mathrm{CMBs}$ is associated with the development of new CMBs after CAS. A further prospective study with a larger sample size is intended to confirm these results.

\section{Disclosure Statement}

The authors report no conflicts of interest.

\section{References}

-1 Fazekas F, Kleinert R, Roob G, et al: Histopathologic analysis of foci of signal loss on gradient-echo $\mathrm{T} 2{ }^{*}$-weighted MR images in patients with spontaneous intracerebral hemorrhage: evidence of microangiopathy-related microbleeds. AJNR Am J Neuroradiol 1999;20:637-642.

-2 Greenberg SM, Vernooij MW, Cordonnier C, Viswanathan A: Cerebral microbleeds: a guide to detection and interpretation. Lancet Neurol 2009;8:165-174.

-3 Shoamanesh A, Kwok CS, Benavente O: Cerebral microbleeds: histopathological correlation of neuroimaging. Cerebrovasc Dis 2011;32:528-534.

-4 Fan YH, Zhang L, Lam WW, Mok VC, Wong KS: Cerebral microbleeds as a risk factor for subsequent intracerebral hemorrhages among patients with acute ischemic stroke. Stroke 2003;34:2459-2462.

-5 Jeon SB, Kang DW, Cho AH, et al: Initial microbleeds at MR imaging can predict recurrent intracerebral hemorrhage. J Neurol 2007;254:508-512.

-6 Greenberg SM, Eng JA, Ning M, Smith EE, Rosand J: Hemorrhage burden predicts recurrent intracerebral hemorrhage after lobar hemorrhage. Stroke 2004;35:1415-1420.

-7 Okazaki S, Sakaguchi M, Hyun B, et al: Cerebral microbleeds predict impending intracranial hemorrhage in infective endocarditis. Cerebrovasc Dis 2011;32:483-488.

8 Jeon SB, Kwon SU, Cho AH, Yun SC, Kim JS, Kang DW: Rapid appearance of new cerebral microbleeds after acute ischemic stroke. Neurology 2009;73:1638-1644.

-9 Sundt TM, Sandok BA, Whisnant JP: Carotid endarterectomy. Complications and preoperative assessment of risk. Mayo Clin Proc 1975;50:301-306.

-10 Archie JP Jr: Carotid endarterectomy with reconstruction techniques tailored to operative findings. J Vasc Surg 1993;17:141-149.

-11 Ogasawara K, Sakai N, Kuroiwa T, et al: Intracranial hemorrhage associated with cerebral hyperperfusion syndrome following carotid endarterectomy and carotid artery stenting: retrospective review of 4,494 patients. J Neurosurg 2007;107:1130-1136.

-12 Piepgras DG, Morgan MK, Sundt TM Jr, Yanagihara T, Mussman LM: Intracerebral hemorrhage after carotid endarterectomy. J Neurosurg 1988;68:532-536. 
-13 Solomon RA, Loftus CM, Quest DO, Correll JW: Incidence and etiology of intracerebral hemorrhage following carotid endarterectomy. J Neurosurg 1986;64:29-34.

-14 Sundt TM Jr, Sharbrough FW, Piepgras DG, Kearns TP, Messick JM Jr, O'Fallon WM: Correlation of cerebral blood flow and electroencephalographic changes during carotid endarterectomy: with results of surgery and hemodynamics of cerebral ischemia. Mayo Clin Proc 1981;56:533-543.

-15 Abou-Chebl A, Yadav JS, Reginelli JP, Bajzer C, Bhatt D, Krieger DW: Intracranial hemorrhage and hyperperfusion syndrome following carotid artery stenting: risk factors, prevention, and treatment. J Am Coll Cardiol 2004;43:1596-1601.

16 Pomposelli FB, Lamparello PJ, Riles TS, Craighead CC, Giangola G, Imparato AM: Intracranial hemorrhage after carotid endarterectomy. J Vasc Surg 1988;7:248-255.

- 17 Schroeder T, Sillesen H, Boesen J, Laursen H, Sorensen P: Intracerebral haemorrhage after carotid endarterectomy. Eur J Vasc Surg 1987;1:51-60.

18 Beneficial effect of carotid endarterectomy in symptomatic patients with high-grade carotid stenosis. North American Symptomatic Carotid Endarterectomy Trial Collaborators. N Engl J Med 1991;325: 445-453.

19 Matsumoto S, Nakahara I, Higashi T, et al: Near-infrared spectroscopy in carotid artery stenting predicts cerebral hyperperfusion syndrome. Neurology 2009;72:1512-1518.

-20 Cordonnier C, Al-Shahi Salman R, Wardlaw J: Spontaneous brain microbleeds: systematic review, subgroup analyses and standards for study design and reporting. Brain 2007;130:1988-2003.

-21 Lee JH, Park KY, Shin JH, et al: Symptomatic hemorrhagic transformation and its predictors in acute ischemic stroke with atrial fibrillation. Eur Neurol 2010;64:193-200.

-22 Tanne D, Kasner SE, Demchuk AM, et al: Markers of increased risk of intracerebral hemorrhage after intravenous recombinant tissue plasminogen activator therapy for acute ischemic stroke in clinical practice: the Multicenter rt-PA Stroke Survey. Circulation 2002;105:1679-1685.

-23 American College of Cardiology Foundation; American Society of Interventional and Therapeutic Neuroradiology; Society for Cardiovascular Angiography and Interventions; Society for Vascular Medicine and Biology; Society of Interventional Radiology; Bates ER, Babb JD, Casey DE Jr, et al: ACCF/SCAI/SVMB/SIR/ASITN 2007 clinical expert consensus document on carotid stenting: a report of the American College of Cardiology Foundation Task Force on Clinical Expert Consensus Documents (ACCF/SCAI/SVMB/SIR/ASITN Clinical Expert Consensus Document Committee on Carotid Stenting). J Am Coll Cardiol 2007;49:126-170.

24 Scheid R, Ott DV, Roth H, Schroeter ML, von Cramon DY: Comparative magnetic resonance imaging at 1.5 and 3 Tesla for the evaluation of traumatic microbleeds. J Neurotrauma 2007;24:1811-1816. 